\title{
Study on the secondary structure and B-cell epitopes of Mycoplasma suis ORF9 protein by Bioinformatics
}

\author{
Si Shan ${ }^{1}$, Nie Peng ${ }^{1}$, and Xiaojun Yan ${ }^{1}$, Caifeng $\mathrm{Ba}^{2}$ \\ ${ }^{1}$ Jiangxi University of Traditional Chinese Medicine, Nanchang, China \\ ${ }^{2}$ Liaoning Medical University, Jinzhou, China
}

Keywords: M suis; ORF9; secondary structure; B-cell epitopes, Bioinformatics

\begin{abstract}
Objective To analyze the spatial structure and structure feature, then predict B-cell epitopes of Mycoplasma suis ORF9 encoding protein. Methods The secondary structure, structural feature and B-cell epitopes were analyzed and predicted by DNAStar software and IDEB. Results the secondary structure of Mycoplasma suis ORF9 protein was relatively regular, in which the potential B cell antigenic epitopes were located at TPKRISGKET, GRESSRLT. Conclusion these valuable information may provide a new method and a new idea for the study of M.suis immunogenicity and pathogenic microorganisms.
\end{abstract}

\section{Introduction}

With the rapid development of bioinformatics, structure and epitopes analysis have become increasingly important. Mycoplasma suis (M. suis) is an uncultivable bacterium lacking a cell wall that attaches to and may invade the red blood cells of pigs (Hoelzle LE, 2014). To date, there were over 30 contries reports about M.suis infection (Wu Yimiao, 2013), M. Suis is severely harmful to human health and animal husbandry. However, no in vitro cultivation systems for M.suis have been available, resulting in little information about biological characteristics and pathogenesis of $\mathrm{M}$. suis infection (Hoelzle LE, 2011).

Hoelzle and his colleagues proposed DNA sequences signed AJ504999 of M. suis contained 11 ORFs, whereas was not carry on researching the biological information and Immunology. According to the literature and the authors' results, it is pointed out that the B cell dominant epitope of proteins were depended on the spatial structure, hydrophilic, flexibility, antigen index and surface accessibility (OU Qin, 2007, SHAN Si, 2013). Epitopes which also known as antigenic determinants are chemical groups that determine the specificity of the antigen.

The study is to predict the spatial structure and B-cell epitopes of M suis ORF9 protein by bioinformatics methods. The secondary structure, hydrophilicity, flexible region, antigenic index and surface probability were analyzed and predicted by the protean module in DNAStar software and B Cell Epitope Prediction Tools of IDEB, then B-cell epitopes were predicted by aggregate analysis. These valuable information may provide a new method for the study of $M$. suis immunogenicity.

\section{Materials and methods}

\subsection{Findimg data of AJ504999 sequence and its ORFs}

Logged into National Center for Biotechnology Information (NCBI) Database, then chose Nucleotide Database, entered accession number “AJ504999”. Aquired data of AJ504999 nucleotide sequence and its protein products are available and reliable.

\subsection{Prediction of the secondary structure}

Opened Editseq module of DNAStar software, then saved amino acid sequence of M. Suis ORF9, finally analyzed and predicted the secondary structure and physicochemical characteristics by Protean module. Whereafter logged into Immune Epitope Database and Analysis resource (IEDB), predicted Average Antigenic Index though Kolaskar-tongaonkar of B Cell Epitope Prediction Tools. 


\subsection{Prediction of epitopes}

The B cell dominant epitope of M. Suis ORF9 encoding proteins were analyzed and predicted by considered parameters as the spatial structure, hydrophilic, flexibility, antigen index and surface accessibility.

\section{Results}

\subsection{Amino acid sequence of M.suis AJ504999-ORF9 as follows:}

ACKGRESSRLTPANIRS. There were 79 AA, and its relative molecular weight was $9111.54 \mathrm{~m}$. w., isoelectric point was 9.23.

\subsection{Prediction of the secondary structure:}

The results of the secondary structure of M.suis ORF9 protein which were analyzed by GamierRobson and Chou-Fasma method showed, the coincidence rate of $\beta$-turn relatively high. There was no $\beta$-sheet in result which analyzed by Chou-Fasman method, moreover, the random coil regions were scattered.(Table.1).

Table 1. Secondary structure of ORF9 protein predicted by methods of Gamier-Robson and ChouFasman

\begin{tabular}{|c|c|c|}
\hline \multirow{2}{*}{$\begin{array}{l}\text { Secondary } \\
\text { structures }\end{array}$} & \multicolumn{2}{|c|}{ regions } \\
\hline & Gamier-Robson & Chou-Fasman \\
\hline$\alpha$-helix & $3-6$ & $1-17$ \\
\hline$\beta$-sheet & $1-2,13-15,60$ & 0 \\
\hline$\beta$-turn & $\begin{array}{c}\text { 7-10,18-23,25-33,35-42,45- } \\
47,51-52,55,56-59,61-71,75,78- \\
79\end{array}$ & $\begin{array}{c}24-31,40-47,49-56,64-67,69- \\
72,74-77\end{array}$ \\
\hline $\begin{array}{l}\text { random } \\
\text { coil }\end{array}$ & $\begin{array}{c}11-12,16-17,24,34,40,43-44,48- \\
50,53-54,72-74,76-77\end{array}$ & - \\
\hline
\end{tabular}

\subsection{Structural features of M.suis ORF9}

Hydrophilicity analysis. The results of hydrophilcity of M.suis ORF9 protein which were analyzed by Kyte-Doolittle method of Protean module showed, there were so much the Hydrophilic segments in the plot such as 2,4,5-9,18,20-23,35-61,63-79aa(Fig. 2 ).

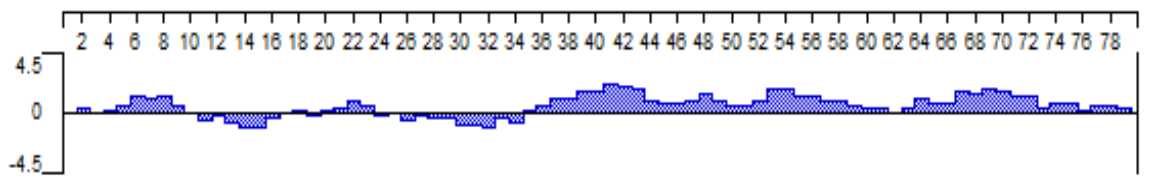

Figure 2. Hydrophilicity plot for the protein of ORF9 analysis by method of Kyte-Doolittle

Flexible analysis. The results of Flexible of M.suis ORF9 protein which were analyzed by Karplus-Schulz method of Protean module showed, there were so much the Flexible regions in the plot such as 8-10,25,39-41,43-60,65-76aa(Fig. 3 ).

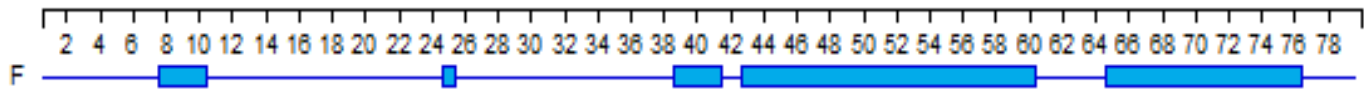

Figure 3. Flexible regions of ORF9 protein predicted by method of Karplus-Schulz

Antigenic index analysis. The results of Antigenic index were analyzed by Jameson-Wolf method of Protean module showed, there were high Antigenic index regions in the plot such as 78,27-28,38-43,47-59,63-79aa( Fig. 4). Besides, the results of Epitope index were analyzed by Kolaskar-Tongaonkar method of IEDB showed, its average Antigenic index was 1.030 ( Fig 5 ). 


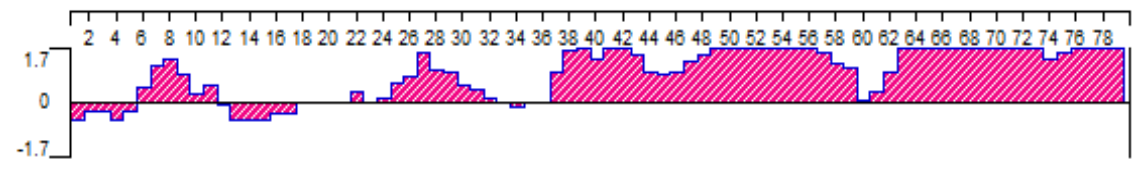

Figure 4. Antigenic index for protein of ORF9

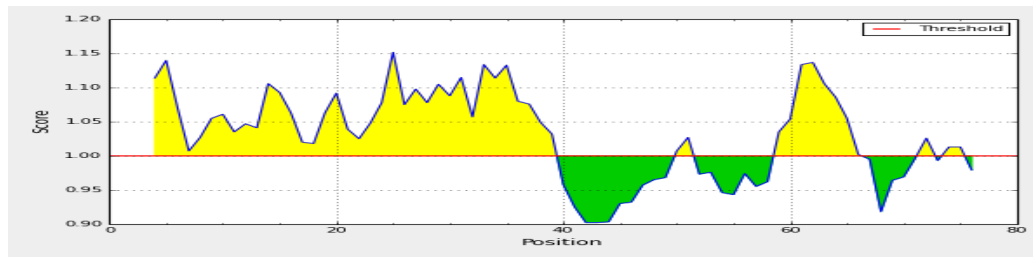

Figure 5. Epitope index for protein of ORF9

Surface probability analysis. The results of Surface probability were analyzed by Emini method of Protean module showed, there were high Surface probability regions in the plot such as 6-8,3842,49-58,66-73,75-76,78aa ( Fig. 6).

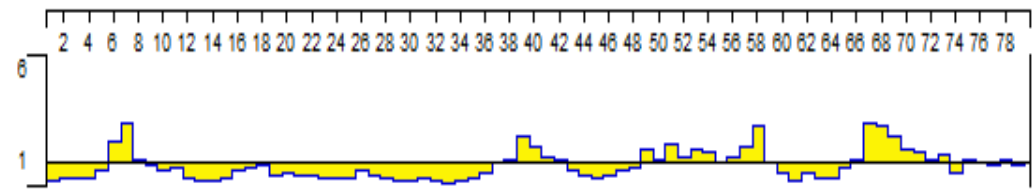

Figure 6. Surface probability plot for the protein of ORF9

\subsection{Prediction of $B$ cell antigenic epitope}

The results showed, 2 segment which suitable for B cell dominant epitope of M. Suis ORF9 proteins through considering parameters as the spatial structure and structural features .The segments which met Surface probability $\geq 1$, Hydrophilicity index $\geq 0$, Antigenic index $\geq 1.030$, and Flexible regions were inside or surrounding, the number of amino acid $\geq 7$, probably should be dominant epitope (Tab.2 ) .

Table 2. Amino acid sequence of B cell antigenic epitope of protein encoded by ORF9

\begin{tabular}{cccc}
\hline $\begin{array}{c}\text { Start } \\
\text { positi } \\
\text { on }\end{array}$ & $\begin{array}{c}\text { End } \\
\text { positio } \\
\mathrm{n}\end{array}$ & $\begin{array}{c}\text { Amino } \\
\text { acid } \\
\text { sequence }\end{array}$ & $\begin{array}{c}\text { The length of } \\
\text { amino acid } \\
\text { sequence }\end{array}$ \\
\hline 49 & 59 & $\begin{array}{c}\text { TPKRIS } \\
\text { GKET } \\
\text { GRESSR } \\
\text { LT }\end{array}$ & 10 \\
66 & 73 & 8 \\
\hline
\end{tabular}

\section{Discussion}

The further study of basic theory and practical application on B cell antigen epitope which was composed of continuous peptide chain of amino acids ,was beneficial to promote the immune recognition, the diagnosis of diseases, fixed-point modification protein molecules, reduce immunogenicity protein drugs, non-toxic side effects of artificial design vaccine and immune intervention (Wu Jing, 2000). Scholars proposed protein spatial structure determines the function of the protein and the distribution of B cell antigen epitope. $\beta$-turn structure were more likely to be B cell antigen epitope which located on the surface of antigen protein so that more conducive to reaction with the chimeric antibody. And there were a far less chance that $\alpha$-helix, $\beta$-sheet were $B$ cell antigen epitope whose structure were stability so that difficult to touch with antibody. In other words, most of B cell antigen epitope located on the surface of antigen protein, so hydrophilic amino acid residue segment and higher surface accessibility index segion which located surfurce of protein were high rate. Otherwise, the flexible region and antigen index of protein determined the 
distribution of B cell antigen epitope. Scholars proposed different analysis methods for B cell antigen epitope prediction. The comprehensive analysis which predicted B cell antigen epitope through synthesized structure parameter such asspatial structure, hydrophilic, flexibility, surface accessibility, antigen index (Song J, 2012, Kyte J, 1982, Xue X, 2011, Sweredoski MJ, 2009). Due to software analysis has certain limitation, synthesized results of variety analysis methods and multiple parameters, which can improve the prediction accuracy and credibility (LIANG Jin, 2009).

\section{Conclusions}

Our study proposed that the secondary structure of M suis ORF9 protein was relatively regular, the potential B cell antigenic epitopes were located at TPKRISGKET. These findings shed new lights on application of vaccines for prevention and treatment of $\mathrm{M}$ suis infecton.

\section{Acknowledgements}

This project was supported by a grant from Liaoning Province technology foundation (No. 20064080026).

\section{References}

[1]Hoelzle LE,Zeder M,Felder KM,et al. Pathobiol ogy of Mycoplasma suis.[J] . Vet J. 2014 Oct; 202 (1):20-25.

[2]Hoelzle LE, Felder KM, Hoelzle K.Porcine eperythrozoonosis: from Eperythrozoon suis to Mycoplasma suis [J].Tierarztl Prax Ausg G Grosstiere Nutztiere. 2011; 39 (4):215-20.

[3]Hoelzle LE, Adelt D, Hoelzle K, et al. Mycoplasma suis ORF1, ORF2, ORF3, ORF4, ORF5, ORF6, ORF7, ORF8, ORF9, ORF10and ORF11 DNA. Unpublished, 2003, 270: 8057.

[4]Kyte J, Doolittle RF. A Simple method for displaying the hydropathic charactor of a protein [J]. J Mol Biol,1982, 157(1):105-132.

[5]Li H, Hu P. Prediction of the B cell epitopes of hemagglutinin and neuraminidase of human infected avian influenza A H7N9 virus[J].Xi Bao Yu Fen Zi Mian Yi Xue Za Zhi. 2013 Dec; 29(12): 1319-21

[6]LIANG Jin, WANG Jing-fei, Progress in the studies of protein B-cell epitope prediction [J]. Chinese Bulletin of Life Sciences, 2009,21( 2):321-323.

[7]OU Qin, ZHU Shan-li, ZHANG Li-fang. Prediction of the secondary structure and B-cell epitopes of EBV LMP2 [J]. Journal of Wenzhou Medical College, 2007, 37(2): 114-118.

[8]SHAN Si, BA Cai-feng. Prediction of secondary structure and B-cell antigenic epitopes of Mycoplasma suis ORF2 protein [J]. Chin J Biologicals, 2013, 26(2):218-211

[9]Song J, He QF. Bioinformatics analysis of the structure and linear B-cell epitopes of aquaporin-3 from Schistosoma japonicum[J]. Asian Pac J Trop Med. 2012, 5(2):107-109. DOI: 10. 1016 /S1995-7645(12)60005-4.

[10]Sweredoski MJ, Baldi P. A novel system for predicting continuous B-cell epitopes [J]. Protein Eng Des Sel. 2009, 22(3):113-120. DOI: 10.1093 /protein/gzn075.

WU Yi-miao.Diagnosis and treatment of Eperythrozoonosis in one case of piglet [J] . Fujian Journal of Animal Husbandry and Veterinary Medicine, 2013, 35(5): 67-68. (in Chinese)

Wu Jing, Wu Wutong. Research Progress on the method of B cell protein antigen epitope [J]. Pharmaceutical Biotechnology, 2000, 7(4): 239-24.

Xue X, Zhu S, Li W, et al.Identification and characterization of novel B-cell epitopes within EBV latent membrane protein 2 (LMP2)[J].Viral Immunol. 2011, 24(3):227-236 